\title{
Ferromagnetic Behavior in Zinc Ferrite Nanoparticles Synthesized using Coprecipitation Technique
}

\author{
Berhanu $\mathrm{H}^{1}$, Raghavender A. $\mathrm{T}^{1^{\star}}$, Kebede $\mathrm{L}^{1}$ and Anjaneyulu $\mathrm{T}^{2}$ \\ ${ }^{1}$ Department of Physics, College of Natural and Computational Sciences, Wollega University, \\ Post Box No: 395, Nekemte, Ethiopia
}

${ }^{2}$ Department of Physics, Narasaraopet Engineering College, Narasaraopet - 522 601, Andhra Pradesh, India

\begin{tabular}{|c|c|}
\hline Abstract & \\
\hline \multirow{12}{*}{ 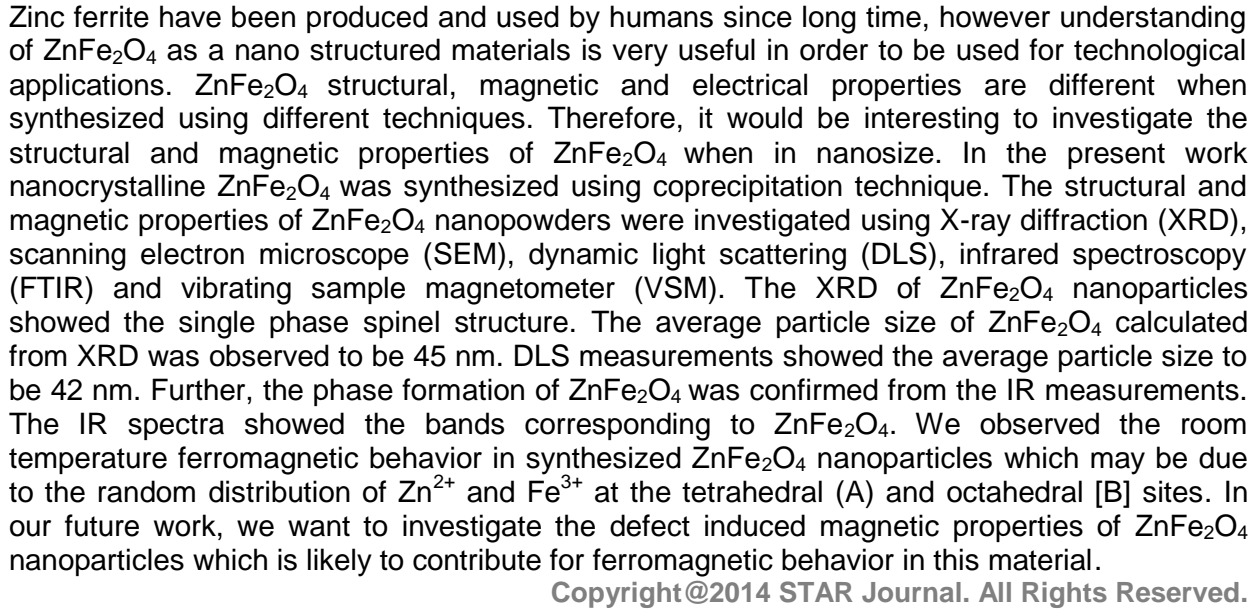 } & Received : 05-10 \\
\hline & Revised \\
\hline & Accepted \\
\hline & \\
\hline & \\
\hline & $\mathrm{Znt}$ \\
\hline & \\
\hline & Mas \\
\hline & on \\
\hline & $\mathbf{T}$ \\
\hline & \\
\hline & \\
\hline
\end{tabular}

\section{INTRODUCTION}

Nanoferrites are being extensively investigated due to their potential applications in magnetic refrigeration, drug delivery, high-density information storage, magnetic fluids etc. (Habibi et al., 2013, Choi et al., 2008). Spinel ferrites have been intensively investigated due to their versatile physical and chemical properties and due to their technological applications in magnetic sensors, biosensors, photocatalysts, nanoelectronics, biomedical (Ling et al., 2013, Durka Prasad et al., 2014). Zinc ferrite is one of the most important technological material having applications in radio engineering, radio technology, semiconductors etc. Bulk zinc ferrite have the normal spinel structure with $\mathrm{Zn}^{2+}$ ions occupying tetrahedral $(\mathrm{A})$ site and $\mathrm{Fe}^{3+}$ ions being occupied in octahedral (B) site and is antiferromagnetic material having Neel temperature around $10 \mathrm{~K}$ (Pettit et al., 1971). The magnetic properties of zinc ferrites significantly depend when a small amount of $\mathrm{Fe}^{3+}$ ions occupy A site (Choi et al., 2008).

The magnetic properties of zinc ferrite nanoparticles was observed to have higher magnetization values compared to the bulk materials and also the synthesis technique might also play important role in achieving the ferromagnetic nature of zinc ferrite (Chen et al., 1980 Singh et al., 2010 and Thirupathi et al., 2012). Several reports on $\mathrm{ZnFe}_{2} \mathrm{O}_{4}$ have showed that, $\mathrm{ZnFe}_{2} \mathrm{O}_{4}$ is paramagnetic at room temperature with Néel temperature $\mathrm{T}_{\mathrm{N}} \approx 10 \mathrm{~K}$. But, recently when $\mathrm{ZnFe}_{2} \mathrm{O}_{4}$ synthesized by different techniques and due to creation of oxygen vacancies ferromagnetic behavior was observed. Based on the above interesting properties of $\mathrm{ZnFe}_{2} \mathrm{O}_{4}$ nanoparticles, in this work we aimed to achieve room temperature ferromagnetic behavior of $\mathrm{ZnFe}_{2} \mathrm{O}_{4}$ nanoparticles synthesized using coprecipitation technique.

\section{MATERIALS AND METHODS}

$\mathrm{ZnFe}_{2} \mathrm{O}_{4}$ nanoparticles were synthesized using coprecipitation method (Raghavender et al., 2011). The AR grade sodium hydroxide $(\mathrm{NaOH})$, zinc (II) nitrate hydrate $\left(\mathrm{Zn}\left(\mathrm{NO}_{3}\right)_{2} \cdot 6 \mathrm{H}_{2} \mathrm{O}\right)$, ferric (III) nitrate nonahydrate $\left(\mathrm{Fe}\left(\mathrm{NO}_{3}\right)_{3} .9 \mathrm{H}_{2} \mathrm{O}\right)(98 \%)$ were used as starting materials. The metal nitrates were dissolved together in a minimum amount of deionized water to get a clear solution. In the metal nitrates precursor solution $\mathrm{NaOH}$ solution was added drop by drop under vigorous stirring. The precipitation occurred immediately to change the reaction solution to dark brown. The entire reaction was carried out at $75{ }^{\circ} \mathrm{C}$ for $2 \mathrm{~h}$. The $\mathrm{pH}$ of the solution was varied by $\mathrm{NaOH}$. The precipitate was filtered using a filter paper and 
Berhanu et alo,

then dried at $220{ }^{\circ} \mathrm{C}$ for $3 \mathrm{~h}$ in oven. The structural characterization of zinc ferrite powders was carried out using Philips (France) X-ray diffraction (XRD) system with $\mathrm{Ni}$ filter using $\mathrm{Cu}-\mathrm{Ka}$ radiation (wave length $\lambda=1.54 \mathrm{~A}^{\circ}$ ). The $\mathrm{ZnFe}_{2} \mathrm{O}_{4}$ structure was confirmed by ABB Bomem MB 102 (Canada) infrared (FTIR) spectrometer. The samples were mixed with $\mathrm{KBr}$ and made in the form of pellets for IR transmission measurements. The particle size and morphology was verified using FEI Quanta (USA) FEG 200 High Resolution Scanning Electron Microscope (HR-SEM) and DLS measurements using LS spectrometer (Switzerland). The $\mathrm{ZnFe}_{2} \mathrm{O}_{4}$ powder was made in the form of pellet by applying 5 Tons of pressure and then SEM measurements was carried. For DLS measurements $\mathrm{ZnFe}_{2} \mathrm{O}_{4}$ powder was dissolved in ethanol and a high intensity laser beam was passed on to the dissolved sample to capture the particle size image. Room temperature magnetic properties were investigated using Lakeshore (USA) VSM 7410.

\section{RESULTS AND DISCUSSION}

Figure 1 shows the X-ray diffraction patterns of $\mathrm{ZnFe}_{2} \mathrm{O}_{4}$ nanoparticles. The XRD pattern shows the formation of single phase spinel structure. The lattice constant $a$ from the XRD analysis was observed to be $8.409 \AA$, which is in agreement with the $\mathrm{ZnFe}_{2} \mathrm{O}_{4}$ nanoparticles synthesized by different techniques (Anjaneyulu et al., 2014). The average particle size D was calculated using most intense peak (3 11 1) employing the Scherrer formula (Raghavender 2013).

$$
D=\frac{0.9 \lambda}{\beta \operatorname{Cos} \theta},
$$

where $\beta$ is the angular line width at half maximum intensity and $y$ is the Bragg angle for the actual peak.
Sci. Technol. Arts Res. J., Oct-Dec 2014, 3(4): 85-88

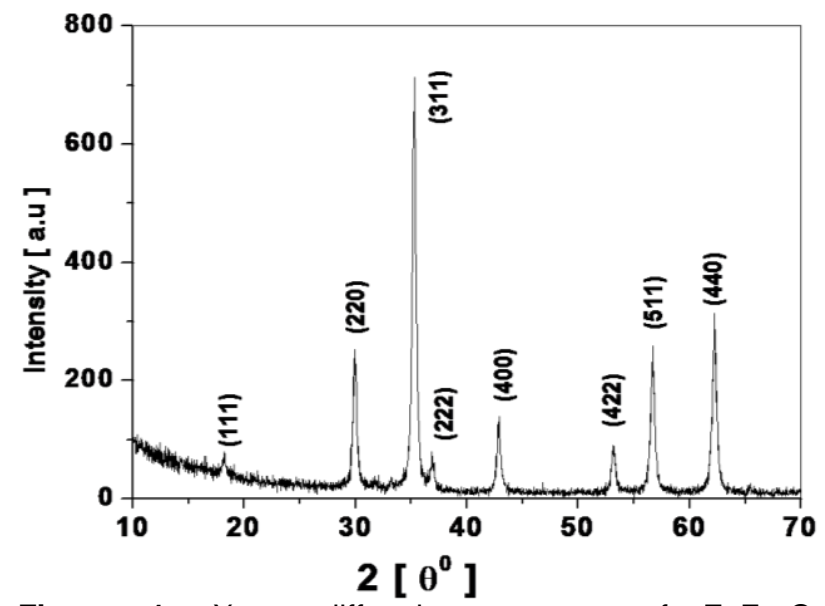

Figure 1: X-ray diffraction patterns of $\mathrm{ZnFe}_{2} \mathrm{O}_{4}$ nanoparticles

The average particle size calculated from XRD spectra was observed to be $45 \mathrm{~nm}$ (Table 1). The particle size was also measured from DLS system. The DLS measurement shows the particle size to be 42 (Figure 2). Therefore the average particle sizes calculated from XRD and DLS measurements are very well in agreement. Figure 3 shows the SEM image of synthesized $\mathrm{ZnFe}_{2} \mathrm{O}_{4}$ nanoparticles. Due to agglomeration in the synthesized nanoparticles we could not clearly observe the particle size distribution. Generally, when the particle size is in nano regime, due to nature and forces between them, agglomeration occurs which seems to be unavoidable when synthesized by few techniques. However, the XRD and DLS measurements support the existence of the nanoparticles in the synthesized $\mathrm{ZnFe}_{2} \mathrm{O}_{4}$ powder.

Table 1: Particle size $D$ from XRD, DLS, lattice constant $a$, IR bands $v_{1}, v_{2}, v_{3}$, Coercivity $H_{c}$, remanence magnetization $M_{r}$, maximum magnetization $\mathrm{M}$ for $\mathrm{ZnFe}_{2} \mathrm{O}_{4}$ nanoparticles

\begin{tabular}{ccccccccc}
\hline $\mathbf{D}_{\text {XRD }}(\mathbf{n m})$ & $\mathbf{D}_{\text {dLS }}(\mathbf{n m})$ & $\mathbf{a}(\AA)$ & $\boldsymbol{V}_{\mathbf{1}}$ & $\boldsymbol{V}_{\mathbf{2}}$ & $\boldsymbol{V}_{\mathbf{3}}$ & $\mathbf{H}_{\mathrm{c}}(\mathbf{O e})$ & $\mathbf{M}_{\mathrm{r}}(\mathbf{e m u} / \mathbf{g})$ & $\mathbf{M}(\mathbf{e m u} / \mathbf{g})$ \\
\hline 45 & 42 & 8.409 & 544 & 388 & 329 & 57 & 1.2 & 10.6 \\
\hline
\end{tabular}

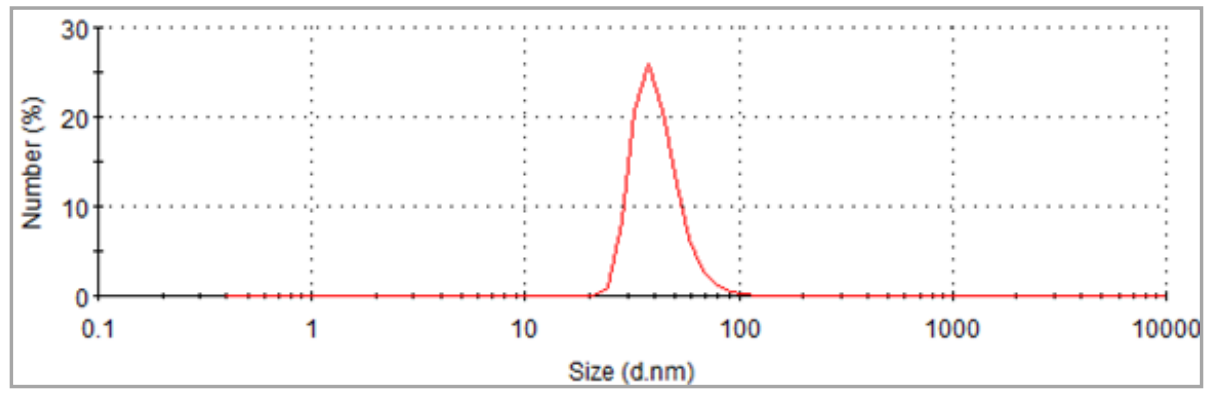

Size distribution by Number

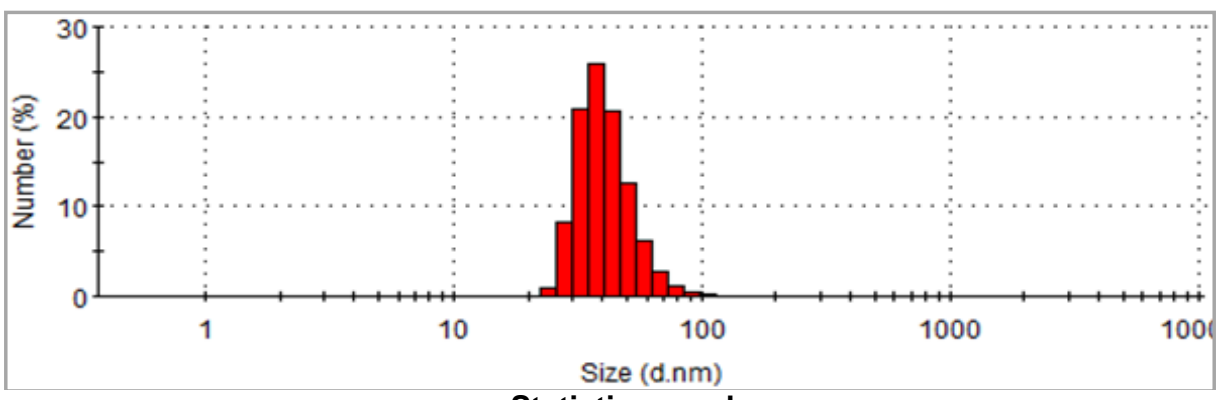

Statistics graph

Figure 2: DLS image by distribution and statistics graph of $\mathrm{ZnFe}_{2} \mathrm{O}_{4}$ nanoparticles. 
Berhanu et al.,

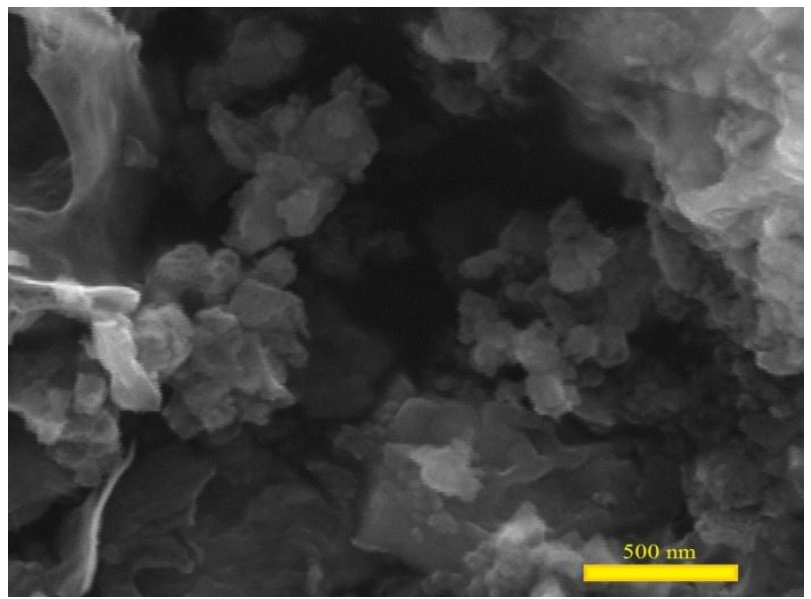

Figure 3: SEM image of $\mathrm{ZnFe}_{2} \mathrm{O}_{4}$ nanoparticles

The IR spectra of $\mathrm{ZnFe}_{2} \mathrm{O}_{4}$ nanoparticles are shown in Figure 4. IR spectra shows the bands corresponding to $\mathrm{ZnFe}_{2} \mathrm{O}_{4}$. The IR spectra shows the three absorption bands. The first band, $v_{1}$, is located in the $800-500 \mathrm{~cm}^{-1}$ range, the second band, $v_{2}$, in the $500-350 \mathrm{~cm}^{-1}$ range, while the third band, $v_{3}$, occurs between 350 and $280 \mathrm{~cm}^{-1}$ range. According to Waldron (Waldron 1955), $v_{1}$ band corresponds to the stretching vibrations of $\mathrm{Zn}^{2+}-\mathrm{O}$ band in tetrahedral sites, while $v_{2}$ is assigned to $\mathrm{Fe}^{3+}$ - O stretching of octahedral sites. On the other hand, $v_{3}$ is result of oscillations of metal atoms in the isotropic force fields of their tetrahedral or octahedral environment. The IR results observed for $\mathrm{ZnFe}_{2} \mathrm{O}_{4}$ are in support with the reported data in the literature (Raghavender et al., 2011; Raghavender 2013; Anjaneyulu et al., 2014).

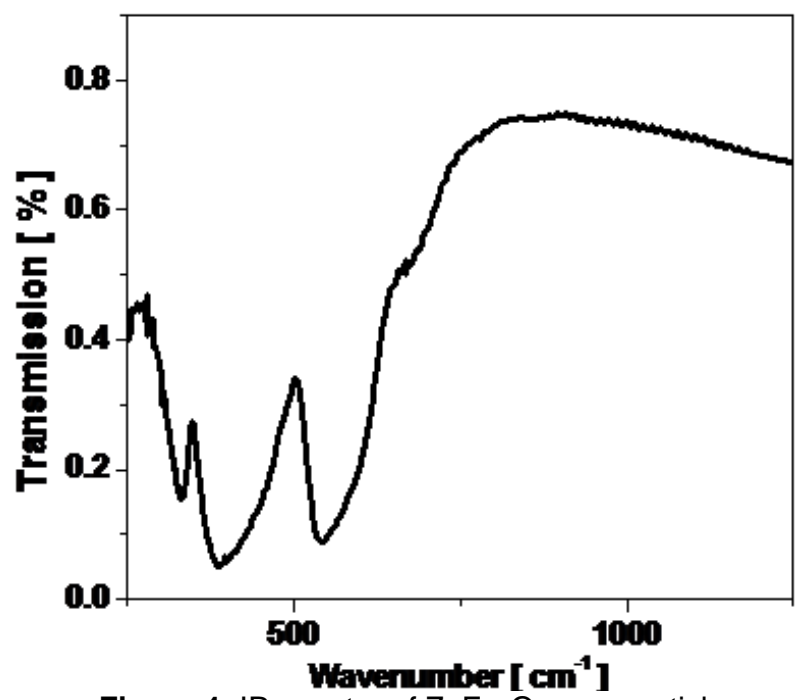

Figure 4: IR spectra of $\mathrm{ZnFe}_{2} \mathrm{O}_{4}$ nanoparticles

Figure 5 shows the room temperature hysteresis curve of $\mathrm{ZnFe}_{2} \mathrm{O}_{4}$ nanoparticles. The derived parameters are listed in Table 1. The hysteresis curve shows ferromagnetic nature of $\mathrm{ZnFe}_{2} \mathrm{O}_{4}$ nanoparticles. The magnetization curve does not seem to be saturated even with the maximum applied field of $10 \mathrm{kOe}$. From the hysteresis curve, the coercivity $\mathrm{H}_{\mathrm{c}}$ was observed to be 57 Oe, remanence magnetization $M_{r}$ was observed to be 1.2 $\mathrm{emu} / \mathrm{g}$ and the maximum magnetization $\mathrm{M}$ to be 10.6 emu/g. $\mathrm{ZnFe}_{2} \mathrm{O}_{4}$ nanoparticles / bulk materials measured at low temperatures or synthesized at low temperatures shows ferromagnetic behavior. Generally, $\mathrm{ZnFe}_{2} \mathrm{O}_{4}$ shows
Sci. Technol. Arts Res. J., Oct-Dec 2014, 3(4): 85-88

the paramagnetic behavior at room temperature as reported in literature. The probable reason for the observed ferromagnetic behavior in our case may be due to several facts such as structural rearrangement, which may induce changes due to the superexchange interaction in the tetrahedral $(A)$ and octahedral $[B]$ sites (Deraz et al., 2012). In the case of $\mathrm{ZnFe}_{2} \mathrm{O}_{4}$ thin films, the random distribution of $\mathrm{Zn}^{2+}$ and $\mathrm{Fe}^{3+}$ at $(\mathrm{A})$ and $[\mathrm{B}]$ sites or the defects causes the ferromagnetic behavior (Raghavender, 2011). In $\mathrm{ZnFe}_{2} \mathrm{O}_{4}$ nanoparticles or bulk materials, the superexchange interaction between $(A)$ and $[B]$ sites does not seem to favor at room temperature, and when measured below room temperature $\mathrm{ZnFe}_{2} \mathrm{O}_{4}$ shows ferromagnetic behavior (Ayyapan et al., 2010; Goya et al., 1999). The ferromagnetic behavior in our case may also be due to nonequilibrium distribution of $\mathrm{Fe}^{3+}$ ions in $(\mathrm{A})$ and [B] sites (Bohra et al., 2006). In $\mathrm{ZnFe}_{2} \mathrm{O}_{4}$ the inversion parameter leads to the ferromagnetic behavior (Hofmann et al., 2004). In fact, the magnetic properties of $\mathrm{ZnFe}_{2} \mathrm{O}_{4}$ are influenced mainly due to the synthesis routes, annealing temperature, cation distribution and the grain size (Deraz et al., 2012). The synthesis route makes a lot of difference in the properties of materials while no difference in some other properties (Upadhyay et al., 2007). The ferromagnetic behavior may also arise due to the spin or structural disorder when $\mathrm{ZnFe}_{2} \mathrm{O}_{4}$ is synthesized by different routes. Therefore, the observed magnetic properties in the present work may be due to the above mentioned facts. In our future work, we will try to investigate in detail the magnetic properties of $\mathrm{ZnFe}_{2} \mathrm{O}_{4}$ nanoparticles prepared under several experimental conditions to clearly understand the origin of ferromagnetic behavior.

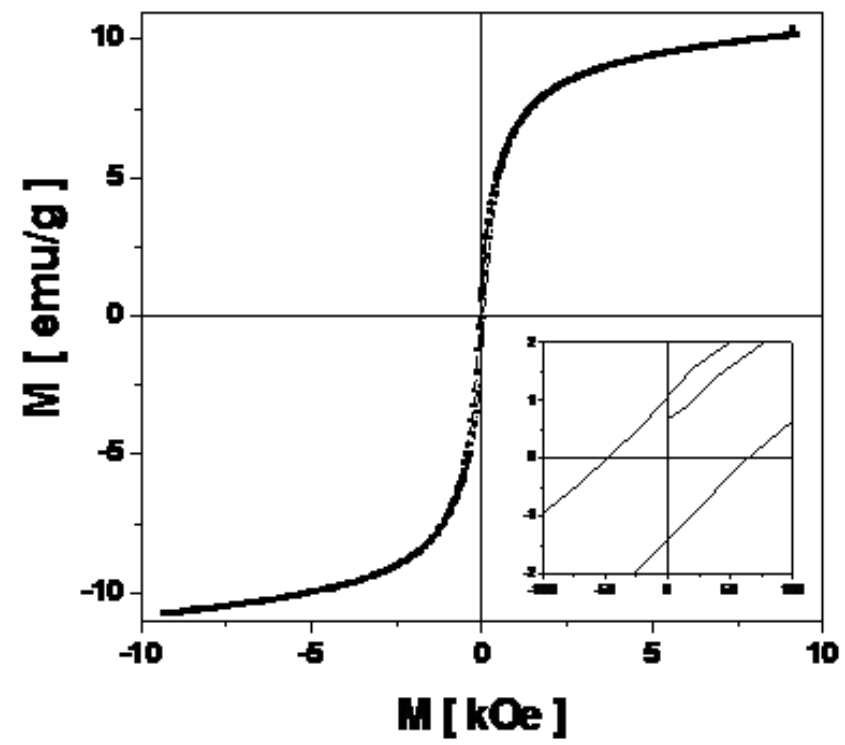

Figure 5: Room temperature $\mathrm{M}-\mathrm{H}$ curve for $\mathrm{ZnFe}_{2} \mathrm{O}_{4}$ nanoparticles. The inset of the figure shows the expanded lower field curve.

\section{CONCLUSION}

$\mathrm{ZnFe}_{2} \mathrm{O}_{4}$ nanoparticles were synthesized using coprecipitation technique. The average particle size of synthesized $\mathrm{ZnFe}_{2} \mathrm{O}_{4}$ were observed to be $45 \mathrm{~nm}$. DLS measurements showed the average particle size around $42 \mathrm{~nm}$. The particle size calculated form XRD and DLS measurements are very well in agreement. The IR spectra showed the bands corresponding to $\mathrm{ZnFe}_{2} \mathrm{O}_{4}$. 


\section{Berhanu et alo,}

Room temperature ferromagnetic behavior was observed for synthesized $\mathrm{ZnFe}_{2} \mathrm{O}_{4}$ nanoparticles. The ferromagnetic behavior in the synthesized $\mathrm{ZnFe}_{2} \mathrm{O}_{4}$ nanoparticles may be due to the random distribution of $\mathrm{Zn}^{2+}$ and $\mathrm{Fe}^{3+}$ at the tetrahedral (A) and octahedral [B] sites, inversion parameter, synthesis techniques etc.

\section{REFERENCES}

Anjaneyulu. T., Raghavender, A.T., Vijaya Kumar, K., Narayana Murthy., Narendra, K. (2014). Effect of particle size on the structural and magnetic properties of nanocrystalline zinc ferrite. Science, Technology and Arts Research Journal 3: 48-51.

Ayyapan, S., Philip Raja, S., Venkateshwaran, C., Philip, J., Raj, B. (2010). Room temperature ferromagnetism in vacuum annealed $\mathrm{ZnFe}_{2} \mathrm{O}_{4}$ nanoparticles. Applied Physics Letters 96:143106.

Bohra, M., Prasad, S., Kumar, N., Misra, D. S., Sahoo, S. C., Venkataramani, N., Krishnana R. (2006). Large room temperature magnetism in nanostructured zin ferrite thin films. Applied Physics Letters 96: 143106.

Chen Q and J.Z. Zhang (1998), Size-dependent superparamagnetic properties of spinel ferrite nanocrystallites. Applied Physics Letters 73: 3156.

Choi E J, Ahn Y and Hahn E J (2008). Size Dependence of the Magnetic Properties in Super paramagnetic ZincFerrite Nanoparticles. Journal of Korean Physical Society 53: 2090

Nagabhushana, H., Thyagarajan, K., Nagabhushana, B.M., Jnaneshwara, D.M., Sharma, S.C., Shivakumara, C., Gopal, N.O., Shyue-Chu Ke. and Chakradhar R.P.S. (2014). Magnetic and dielectric interactions in nano zinc ferrite powder: Prepared by self-sustainable propellant chemistry technique. Journal of Magnetism and Magnetic Materials 358-359: 132-141.

Deraz, N.M., Alarifi, A. (2012). Synthesis and physiochemical properties of nanomagnetic zinc ferrite system. International journal of electrochemical science 7: 37983808 .

Goya, G.F., Rechenberg, H.R. (1999). Ionic disorder and Néel temperature in $\mathrm{ZnFe}_{2} \mathrm{O}_{4}$ nanoparticles. Journal of Magnetism and Magnetic Materials 196-197: 191.
Sci. Technol. Arts Res. J., Oct-Dec 2014, 3(4): 85-88

Habibi M H and Habibi A H (2013), Effect of the thermal treatment conditions on the formation of zinc ferrite nanocomposite, $\mathrm{ZnFe} 2 \mathrm{O} 4$ by sol-gel method. Journal Thermal Analytical Calorimetry 113: 843-847.

Hofmann, M., Campbell, S. J., Ehrhardt, H., Feyerherm, R. (2004). The magnetic behavior of nanostructural zinc ferrite. Journal of Material Science 39: 5057 -5065.

Liang Y C and Hsia H Y (2013), Growth and crystallographic feature-dependent characterization of spinel zinc ferrite thin films by RF sputtering. Nanoscale Research Letters 8: 537.

Pettit G A and Forester D W (1971), Mossbauer study on cobalt zinc ferrite, Physical Review B 4: 3912-3923.

Raghavender, A. T., Nguyen Hoa Hong. (2011). Dependence of Néel temperature on the particle size of $\mathrm{MnFe}_{2} \mathrm{O}_{4}$. Journal of Magnetism and Magnetic Materials 323: 21452147.

Raghavender, A. T., Biliskov, N., Skoko, Z. (2011). XRD and IR analysis of nanocrystalline $\mathrm{Ni}-\mathrm{Zn}$ ferrite synthesized by the sol-gel method. Materials Letters 65: 677 - 680.

Raghavender, A. T. (2013). Synthesis and Characterization of Cobalt Ferrite Nanoparticles. Science, Technology and Arts Research Journal 2: 01-04.

Raghavender, A.T. (2011). Room temperature ferromagnetism in laser ablated $\mathrm{Zn}$ ferrite thin films. Materials Letters 65: 3636-3638.

Singh, J.P., Payal, R.S., Srivastava, R.C., Agarwal, H.M., Prem Chand., Tripathi, A. and Tripath, R.P. (2010). Effect of thermal treatment on the magnetic properties of nanostructured zinc ferrite. Journal of Physics: Conference Series 217: 012108 (1-4).

Thirupathi G and Singh R (2012), Magnetic Properties of Zinc Ferrite Nanoparticles, IEEE Transactions on Magnetics 48: 1-4.

Upadhyay C., Verma H C., Sathe V., Pimpale A.V. (2007). Effect of grain and synthesis routes on the magnetic properties of chemically prepared nanosize $\mathrm{ZnFe}_{2} \mathrm{O}_{4}$. Journal of Magnetism and Magnetic Materials 312: 271279.

Waldron R D (1955), Infrared spectra of ferrites. Physical Review 99: 1727 - 1735. 